\title{
CALIDAD DE VIDA EN FAMILIAS CON PERSONAS CON SÍNDROME DE ANGELMAN
}

\author{
Esther Moraleda Sepúlveda \\ Facultad de Ciencias de la Salud \\ Universidad de Castilla-La Mancha \\ Patricia López Resa \\ Facultad de Ciencias de la Salud \\ Universidad de Castilla-La Mancha \\ Soraya Delgado Matute \\ Facultad de Ciencias de la Salud \\ Universidad de Castilla-La Mancha \\ Romina Frontera \\ Facultad de Ciencias de la Salud \\ Universidad de Castilla-La Mancha \\ Estela Cañadas Camacho \\ Facultad de Ciencias de la Salud \\ Universidad de Castilla-La Mancha
}

Recepción Artículo: 10 mayo 2021

Admisión Evaluación: 10 mayo 2021

Informe Evaluador 1: 19 mayo 2021

Informe Evaluador 2: 24 mayo 2021

Aprobación Publicación: 02 junio 2021

\section{RESUMEN}

El Síndrome de Angelman (SA) es una enfermedad rara caracterizada por discapacidad intelectual grave, retraso en el desarrollo, ausencia de habla o habla mínima, microcefalia, ansiedad, disfunciones motoras, marcha atáxica y deficiencias en las relaciones sociales. Sus características hacen que estas personas necesiten de intervención de varios especialistas clínicos y educativos a lo largo de su vida. El objetivo de este estudio fue conocer el nivel de calidad de vida que describían las familias de esta población. La muestra estuvo compuesta por 7 familias con hijos con Síndrome de Angelman de entre 4 y 16 años que fueron evaluadas a través de la Escala Kidslife. Los datos encontrados indician que el grado de dependencia limita ciertas características de la vida de la persona con Síndrome de Angelman y que esto repercute directamente en el nivel de calidad de vida que describen las familias en todos los niveles. Estos resultados nos llevan a analizar las implicaciones sociales y emocionales que pueden tener para las familias y para su entorno.

Palabras claves: síndrome de Angelman; familias, calidad de vida

\section{ABSTRACT}

Quality of life in families with people with angelman syndrome. Angelman Syndrome (AS) is a rare disease characterized by severe intellectual disability, developmental delay, minimal or no speech, microcep- 


\section{CALIDAD DE VIDA EN FAMILIAS CON PERSONAS CON SÍNDROME DE ANGELMAN}

haly, anxiety, motor dysfunction, ataxic gait and impairment in social relationships. Their characteristics mean that these individuals require the intervention of various clinical and educational specialists throughout their lives. The aim of this study was to know the level of quality of life described by the families of this population. The sample was composed of 7 families with children with Angelman Syndrome between 4 and 16 years old who were evaluated through the Kidslife Scale. The data found indicate that the degree of dependence limits certain characteristics of the life of the person with Angelman Syndrome and that this has a direct impact on the level of quality of life described by the families at all levels. These results lead us to analyze the social and emotional implications for the families and their environment.

\section{Keywords: Angelman syndrome; families, quality of life}

\section{INTRODUCCIÓN}

El Síndrome de Angelman (SA en adelante) es un trastorno del neurodesarrollo con un componente base genético que es debido a una mutación en el cromosoma 15q11-13. La primera descripción clínica data de 1965 y fue realizada por el doctor en pediatría Harry Angelman, quien describió tres casos de niños con características cognitivas, comportamentales y físicas semejantes. Entre ellas se encontraban el retraso mental, ataxia, risa fácil, convulsiones, microcefalia y ausencia de lenguaje (Navarro y Julianes, 2005). El patrón conductual del síndrome es caracterizado por ataques de risa injustificados, apariencia feliz, interés por el agua, personalidad fácilmente excitable e hiperactividad. Bower y Jeavons (1967) lo denominaron como "happy puppet syndrome", debido a las características físicas descritas, el término no fue aceptado por los familiares por lo que terminó por denominarse Síndrome de Angelman, AS en adelante, aludiendo así a su descubridor.

La prevalencia de este síndrome es de 1 caso por cada 15.000 recién nacidos y puede afectar de igual modo, a hombres como a mujeres. El diagnóstico es considerado complejo ya que se trata de un síndrome relativamente joven, con probabilidad de que existan muchos casos sin diagnosticar o posiblemente con un falso diagnóstico. No obstante, pese a que la prevalencia real del síndrome es desconocida, podría estar en torno a 1/15.000 (Williams, 2009).

El AS no se suele identificar con frecuencia en recién nacidos, debido a que las dificultades en el desarrollo que lo caracterizan no son plenamente específicas en los primeros meses, si no que los indicios de estas comienzan a visibilizarse entre los 6 y los 12 meses de edad, no siendo hasta los 3 años cuando se presenta la posibilidad de realizar un diagnóstico. Según Brun y Artigas (2005) en la actualidad, en España, debido a la optimización de las pruebas de diagnóstico y al amplio conocimiento del síndrome, el diagnóstico se podría obtener en edades más tempranas.

Por otro lado, y según Brun (2010) se podrían agrupar las principales características del Síndrome de Angelman en cuatro campos:

En primer lugar, las características cognitivas que afectan SA son el retraso mental profundo, trastorno del lenguaje, falta de atención e hiperactividad. Cabe destacar que, las personas con SA dependen de métodos de comunicación asistida o no, los métodos que no son asistidos van a incluir vocalizaciones no verbales, gestos naturales y signos. Sin lugar a duda, las personas con SA podrían comunicarse completamente con la participación de la familia, y sin olvidar que el uso de SAAC repercutirá en mejorar la calidad de vida de estos pacientes (Calculator, 2010).

En segundo lugar, las características físicas que contempla el SA son la microcefalia, boca grande, separación entre los dientes, hipopigmentación cutánea, escoliosis, pelo y ojos claros, macroglosia y prognatismo.

En tercer lugar, las características conductuales que acompañan al síndrome se caracterizan por una constante apariencia de felicidad y risa fácil, personalidad excitable, aleteo de manos, hiperactividad, dificultades para mantener la atención, ansiedad, agresividad, y alta resistencia al dolor.

En último lugar y no menos importante, las características neurológicas que afectan al síndrome son la diplejía espástica, que puede ir de leve a moderada, ataxia, temblores, movimientos espasmódicos y epilepsia; Esta última es una de las principales características de este síndrome ya que lo suele presentar un 80\% de los pacien- 
tes. Por otro lado, también existe un gran porcentaje de pacientes, en torno al 40 y $80 \%$, que presentan problemas para conciliar y mantener el sueño (Guitart et al., 2015).

Las investigaciones actuales tienden a explorar el entorno cultural y familiar de las personas con SA, relacionando así la calidad de vida y las expectativas con las habilidades comunicativas de las personas con SA.

\section{MÉTODO}

\section{Participantes}

La muestra estuvo compuesta por 7 familias, residentes en distintas áreas del territorio Español, con hijos diagnosticados de Síndrome de Angelman y discapacidad intelectual de moderada a profunda. Se incluyeron 6 madres y un padre, cuatro de ellos con niños entre 6 y los 12 años de edad y las tres familias restantes con adolescentes entre los 13 y los 20 años.

\section{Instrumento}

Cada familia fue evaluada mediante la Escala KidsLife. Este método es utilizado para valorar la calidad de vida de niños y adolescentes de entre 4 y 21 años de edad con algún tipo de discapacidad intelectual (Mora, Salamanca, Córdoba, y Gómez, 2020). Está compuesta de un total de 96 cuestiones relativas a la calidad de vida que pueden ser respondidas por un observador externo que conoce bien al sujeto evaluado. La Escala KidsLife proporciona puntuaciones estándar y percentiles para 8 aspectos distintos de la calidad de vida: Inclusión social; Autodeterminación; Bienestar emocional; Bienestar físico; Bienestar material; Derechos; Desarrollo Personal y Relaciones Interpersonales.

\section{Procedimiento}

Para la realización de este estudio se contactó con la Asociación de Síndrome de Angelman por medio de correo electrónico y redes sociales, se informó de la intención que tenía el trabajo y de si querían formar parte de este. Posteriormente se les indicó la política de privacidad de datos a las familias pertenecientes a la asociación que quisieron colaborar, y se les pasó el consentimiento informado para autorizar el uso de esos datos. Una vez realizado este primer proceso se les envió el cuestionario de forma telemática en formato google forms pudiéndose rellenar desde cualquier dispositivo electrónico, con una duración de unos 20-30 minutos aproximadamente.

\section{Análisis de datos}

Los resultados fueron analizados en base a tres variables consideradas en la Escala Kids Life: nivel de dependencia (moderada, severa y alta); nivel de necesidad (limitado, intermitente, extenso y generalizado) y nivel de discapacidad (leve, moderada, grave y profunda). Se comprobó la normalidad dela muestra mediante la prueba de Kolmogorov-Smirnoff, resultando ser no paramétrica. Seguidamente, los resultados fueron analizados mediante la prueba de U- Mann Whitney. Para analizar la correlación entre variables se utilizó la prueba Rho de Spearman.

\section{RESULTADOS}

Los datos mostraron diferencias significativas en las puntuaciones estándar en las distintas subáreas que componen la escala $(p<0.005)$ : Inclusión social $(X=11.42, D T=2.63)$, Autodeterminación $(X=8.00, D T=2.94)$, Bienestar emocional $(X=8.71, D T=2.42)$, Bienestar físico $(X=10.85, D T=2.60)$, Bienestar material $(X=8.00$, $\mathrm{DT}=4.28)$, Derechos $(X=8.57, \mathrm{DT}=3.59)$, Desarrollo personal $(X=8.42$, DT $=4.35)$ y Relaciones interpersonales $(X=8.00, \mathrm{DT}=1.21)$.

Los datos evidenciaron diferencias significativas para el nivel de discapacidad en las subáreas de Inclusión social, Bienestar emocional, Bienestar físico, Derechos, Desarrollo Personal y Relaciones interpersonales ( $p<$ 0.05) entre los participantes con SA y un grado moderado de discapacidad y entre aquellos con un grado severo de discapacidad. Así mismo, también se encontraron diferencias estadísticamente significativas en las subáreas 


\section{CALIDAD DE VIDA EN FAMILIAS CON PERSONAS CON SÍNDROME DE ANGELMAN}

de Bienestar emocional, Bienestar Material, Derechos y Desarrollo Personal $(P<0.05)$ entre los padres cuyo hijo/a con SA y un grado severo de discapacidad y aquellos cuyos hijos presentaban una discapacidad profunda.

En relación a la necesidad de apoyo se encontraron diferencias significativas entre los sujetos con SA que presentaban una necesidad extensa de apoyos y aquellos que presentaban una necesidad de apoyos generalizada en las áreas de Bienestar emocional y Desarrollo personal $(p<0.05)$.

En lo que respecta al grado de dependencia los resultados evidenciaron diferencias estadísticamente significativas entre los sujetos con un grado severo de dependencia y aquellos que presentaban una gran dependencia en las subáreas de Inclusión social, Bienestar físico y Relaciones interpersonales $(p<0.05)$.

Los resultados también fueron analizados según las edades de los niños, quienes fueron distribuidos en dos grupos de edad: 5 a 10 años y de 11 a 16 años. No se encontraron diferencias estadísticamente significativas entre los grupos de edad para todos los dominios evaluados.

\section{DISCUSIÓN}

Con el presente estudio se ha pretendido analizar la calidad de vida de las familias de las personas con SA, dado que la obtención de un perfil de calidad de vida permite la planificación individualizada de dichos apoyos (Zorrilla, Alonso, Sánchez, Ezquerra y Fernández, 2015). Para ello se ha partido de la concepción más reciente de la discapacidad, entendiéndola como el resultado de la interacción entre la persona y su entorno de manera que la disponibilidad de apoyos favorecerá significativamente el nivel de funcionamiento (Alonso y Schalock, 2010; Puente, Fernández, Alvarado y Jiménez, 2014). Los datos señalan que el grado de discapacidad, el grado de necesidad y el grado de dependencia determinan en gran medida el nivel de calidad de vida familiar, en contra a lo que ocurre en otros estudios evaluados en otro tipo de discapacidades intelectuales, como en el caso del Síndrome de Williams (Moraleda y López, 2021).

Con respecto a las dimensiones de calidad de vida, en el área Inclusión Social los resultados obtenidos ponen de manifiesto que, a pesar de la mayor visibilidad, la inclusión social de este colectivo sigue siendo un tema pendiente en concordancia con las publicaciones de Escudero y Martínez (2011) y Gómez, Verdugo, Arias, Navas y Schalock (2013).

Parece, además, que los resultados en las diferentes dimensiones de calidad de vida muestran unas puntuaciones muy bajas. Por ejemplo, en el área de la autodeterminación, Wehmeyer y Garner (2003) afirman que la toma de decisiones importantes, tales como el centro o la modalidad de escolarización, es llevada a cabo de manera unilateral por los padres durante la infancia y la adolescencia, sin tener en cuenta a la persona con discapacidad intelectual. Los resultados aportados por las familias concuerdan con los de otras investigaciones que reflejan que la toma de decisiones se encuentra significativamente ligada al grado de discapacidad (Smith, Morgan y Davidson, 2005).

En relación a las dimensiones de bienestar emocional, físico y material los resultados obtenidos ponen de manifiesto un grado bajo de satisfacción en estas áreas (Cummis, 2003; Felce 2006). Estos resultados siguen la línea de otras investigaciones que afirman que los problemas de conducta producidos por los desajustes emocionales suelen generar un impacto negativo sobre el bienestar, tanto familiar como individual (Bailey, Golden, Roberts y Ford, 2007; Blacher y Baker, 2007). También en relación a estas categorías, Guyard, Michelsen, Arnaud, Lyons, Cans y Fauconnier (2012) destacan el aumento del gasto económico de las familias de individuos con Discapacidad Intelectual, repercutiendo negativamente en la calidad de vida de las familias.

En la dimensión denominada derechos, de nuevo los resultados aportados por los padres coinciden otros estudios que relacionan esta área con el grado de discapacidad. En esta misma línea Turnbull y Turnbull (2001) añaden que, cuando la discapacidad es ligera o moderada los progenitores tienden a manifestar la escasez de oportunidades y la falta de apoyos para que los individuos con discapacidad intelectual ejerzan sus derechos. Parece, por tanto, que, en cierta medida, las familias siguen percibiendo a sus hijos como indefensos lo que puede Ilevarles en algunos casos a la sobreprotección (Robledo y García, 2007). 
En cuanto al desarrollo personal de estos individuos los datos obtenidos destacan que los padres asumen, en general, que sus hijos se encuentran en un proceso continuo de aprendizaje de habilidades sociales indispensables para el desarrollo personal y, por ende, para una calidad de vida más que satisfactoria (Ramos, 2011).

Por último, la dimensión de las relaciones interpersonales suele ser otra de las dimensiones que más deterioradas se encuentran y es que, por lo general, tanto las personas con discapacidad intelectual como sus familias tienen un menor número de relaciones y, por tanto, son más propensas al riesgo de aislamiento social (Migerode, Maes, Buysse y Brondeel, 2012).

En conclusión, resulta muy difícil, por tanto, homogenizar a todos los individuos que presenten discapacidad intelectual, en este caso SA, y a sus familias, bajo el concepto de calidad de vida pues esta se encuentra altamente influenciada por diversos factores que conforman el bienestar personal (Schalock y Verdugo, 2003; Schalock y Verdugo, 2007; Schalock, 2013; Van Loon, 2013). No obstante, es muy importante tenerlo en cuenta a la hora de ofrecer los apoyos y recursos necesarios para ayudar a las familias durante todo el ciclo vital.

\section{REFERENCIAS BIBLIOGRÁFICAS}

Alonso, M. Á. V., \& Schalock, R. L. (2010). últimos avances en el enfoque y concepción de las personas con dis capacidad intelectual. Revista española sobre discapacidad intelectual, 41(4), 7-21.

Angelman, H. (1965). 'Puppet' children a report on three cases. Developmental Medicine \& Child Neurology, 7(6), 681-688.

Artigas-Pallarés, J., Brun-Gasca, C., Gabau-Vila, E., Guitart-Feliubadaló, M., \& Camprubí-Sánchez, C. (2005). Aspectos médicos y conductuales del síndrome de Angelman. Revista de Neurología (Madrid), 41, 649-56.

Bailey Jr, DB, Golden, RN, Roberts, J. y Ford, A. (2007). Depresión materna y discapacidad del desarrollo: crítica de la investigación. Revisiones de investigaciones sobre retraso mental y discapacidades del desarrollo , 13 (4), 321-329.

Blacher, J. y Baker, BL (2007). Impacto positivo de la discapacidad intelectual en las familias. Revista estadounidense sobre retraso mental, 112 (5), 330-348.

Bower, B. D., \& Jeavons, P. M. (1967). The "happy puppet" syndrome. Archives of disease in childhood, 42(223), 298.

Brun C. \& Artigas J. (2005) Síndrome de Angelman: del gen a la conducta. Ed. Nau Llibres.

Calculator, S. N., \& Black, T. (2010). Parents' priorities for AAC and related instruction for their children with Angelman syndrome. Augmentative and Alternative Communication, 26(1), 30-40.

Cummins, RA y Lau, AL (2003). ¿Integración comunitaria o exposición comunitaria? Una revisión y discusión en relación a personas con discapacidad intelectual. Revista de investigación aplicada en discapacidad intelectual, 16 (2), 145-157.

Escudero, J. M., \& Martínez, B. (2011). Educación inclusiva y cambio escolar. Revista iberoamericana de educación, 55(1), 85-105.

Felce, D. (2006). La calidad de vida en servicios de vida comunitaria: cuestiones sobre la medición subjetiva y objetiva. En Rompiendo inercias: claves para avanzar (pp. 73-88). Amarú.

Galván-Manso, M., Campistol, J., Monros, E., Póo, P., Vernet, A. M., Pineda, M., ... \& Sanmartí, F. X. (2002). Síndrome de Angelman: características físicas y fenotipo conductual en 37 pacientes con diagnóstico genético confirmado. Revista de Neurología (Madrid), 35, 425-9.

Gómez, LE, Verdugo, M. Á., Arias, B., Navas, P., y Schalock, RL (2013). El desarrollo y uso de perfiles de proveedores a nivel organizacional y de sistemas. Evaluación y planificación de programas, 40 , 17-26.

Guitart, M., Camprubí, C., Fernández, C., Gener, B., \& Gabau, E. (2015). Síndrome de Angelman. Enfermedades de Impronta: Guías de buena práctica clínica, 113-146.

Guyard, A., Michelsen, SI, Arnaud, C., Lyons, A., Cans, C. y Fauconnier, J. (2012). Midiendo el concepto de impacto de la discapacidad infantil en los padres: Validación de una medición multidimensional en una población con parálisis cerebral. Investigación en discapacidades del desarrollo, 33 (5), 1594-1604. 


\section{CALIDAD DE VIDA EN FAMILIAS CON PERSONAS CON SÍNDROME DE ANGELMAN}

Migerode, F., Maes, B., Buysse, A., \& Brondeel, R. (2012). Quality of life in adolescents with a disability and their parents: The mediating role of social support and resilience. Journal of Developmental and Physical Disabilities, 24(5), 487-503.

Mora-Antó, A., Salamanca-Duque, L. M., Córdoba-Andrade, L., \& Gómez-Sánchez, L. E. (2020). Estructura Dimensional de la Escala KidsLife, versión Colombia, para la Evaluación de Calidad de Vida en Discapacidad Intelectual. Psykhe (Santiago), 29(1), 1-21. https://dx.doi.org/10.7764/psykhe.29.1.1399

Moraleda Sepúlveda, E., López Resa, P. (2021). Evaluando la calidad de vida en familias con pacientes con Síndrome de Williams. Health and Quality of Life Outcomes, 19, 121 (2021). https://doi.org/10.1186/s12955-021-01704-0

Muñoz-Cabello, B., Rufo-Campos, M., Madruga-Garrido, M., Blanco-Martínez, B., Ruiz-Del Portal, L., \& Candau Fernández-Mensaque, R. (2008). Crisis epilépticas en el síndrome de Angelman. Revista de Neurología, 47(3), 113-118.

Puente Ferreras, A., Fernández Lozano, M. P., Alvarado Izquierdo, J. M., \& Jiménez Rodríguez, V. (2014). Síndrome Williams: una enfermedad rara con sintomatología contradictoria.

Ramos, M. G. (2011). Habilidades sociales en niños y niñas con discapacidad intelectual. Eduinnova.

Robledo, P., \& García, J. (2007). Instrumento de evaluación de las necesidades de las familias con hijos con dificultades del desarrollo: FAOP. JN García (Coor.,), Instrumentos y programas de intervención en las dificultades del desarrollo.

Schalock, R. L., \& Verdugo, M. A. (2003). Calidad de vida. Manual para profesionales de la educación, la salud y servicios sociales. Alianza editorial.

Schalock, R. L., \& Verdugo Alonso, M. Á. (2007). El concepto de calidad de vida en los servicios y apoyos para personas con discapacidad intelectual.

Schalock, R. L., \& Verdugo, M. A. (2013). The transformation of disabilities organizations. Intellectual and Developmental Disabilities, 51(4), 273-286.

Smith, R. B., Morgan, M., \& Davidson, J. (2005). Does the daily choice making of adults with intellectual disability meet the normalisation principle?. Journal of Intellectual and Developmental Disability, 30(4), 226-235.

Turnbull, A. P., \& Turnbull, H. R. (2001). Families, professionals, and exceptionality: Collaborating for empowerment. Prentice Hall.

Van Loon, J. (2013). Un sistema de apoyos centrado en la persona. Mejoras en la calidad de vida a través de los apoyos.

Wehmeyer, M. L., \& Garner, N. W. (2003). The impact of personal characteristics of people with intellectual and developmental disability on self determination and autonomous functioning. Journal of Applied Research in Intellectual Disabilities, 16(4), 255-265.

Williams, C., Peters, S., \& Calculator, S. (2009). Información acerca del síndrome de Angelman. Angelman Fundation [sede web].[citado 14 nov 2011]. Disponible en:

http://www.angelman.org/stay-informed/facts-about-angelman-syndrome-inspanish.

Zorrilla, I. H., Alonso, M. Á. V., Sánchez, L. E. G., Ezquerra, S. F., \& Fernández, P. C. (2015). Evaluación de la calidad de vida en personas con discapacidades significativas: aplicación de la Escala San Martín en la Fundación Obra San Martín. Revista Española de Discapacidad (REDIS), 3(1), 93-105. 